\title{
Fem stress analysis of a barrel reamer
}

\author{
Attila Baksa, Gábor Ladányi, \\ Sándor Szirbik, Zoltán Virág \\ University of Miskolc, Hungary
}

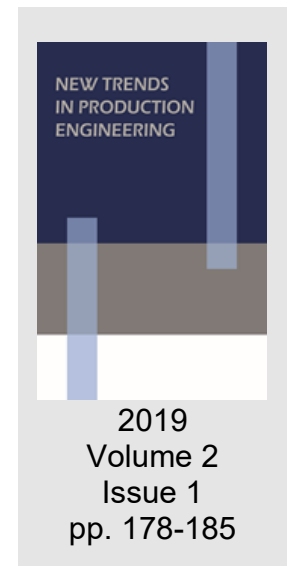

Date of submission to the Editor: 05/2019

Date of acceptance by the Editor: 07/2019

\section{INTRODUCTION}

Horizontal Directional Drilling (Fig. 1) is one of the most versatile technologies for laying down pipes without milling, and is a diversified solution in public utility construction. The trenchless drilling method with only one start and exit point, means several kilometres of pipelines can be laid beneath rivers, cities, mountains and the countryside. It involves the use of a directional drilling machine, and associated attachments, to accurately drill along the chosen bore path and back ream the required pipe (Hair 1991).

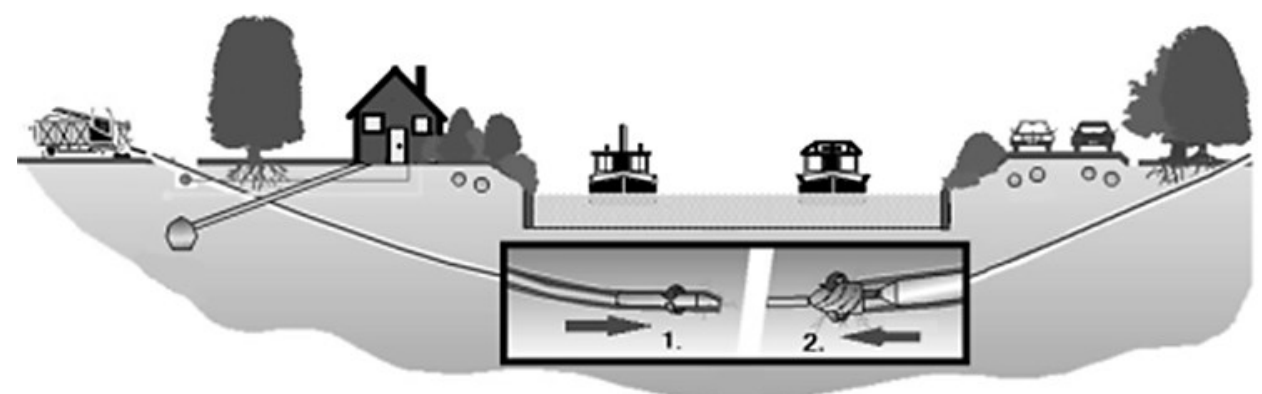

Fig. 1 Horizontal Directional Drilling technology

The trail basically depends on the parameters of the used protective and utility pipes and the operating environment, considering that the minimum inflection radius of the drill shanks needs to be between 25 and $140 \mathrm{~m}$ depending on the type of machinery.

A walk-over tracking system is used to help guide and monitor the location of the bore. The system is comprised of a transmitter and receiver. The transmitter emits a continuous signal, which is picked up by a portable hand-held receiver. Data transmitted to the receiver allows the tracking hand to determine position and depth as well as spatial position of the drill bit. This information allows the operator to track location along the planned bore and to make changes as needed. This follow-up is imperative as there are different reasons, such as the change of geological conditions - due to the greatest attention, the drill bit can 
be diverted from the pre-planned direction. Such an operating failure can cause serious problems in a well-publicized environment.

The first stage consists of drilling a small diameter pilot hole. Drilling fluid (mud) is pumped through the drill pipe to the drill bit where high pressure jets and the bit will grind the soils ahead of the drill stem. The drilling fluid will also carry the cuttings back to the entrance pit at the drill rig. The speed of the pilot hole will vary upon existing soil conditions and the amount of steering which is required. Upon reaching the exit point, the beacon housing and bit is detached and replaced with a reamer (Fig. 2).

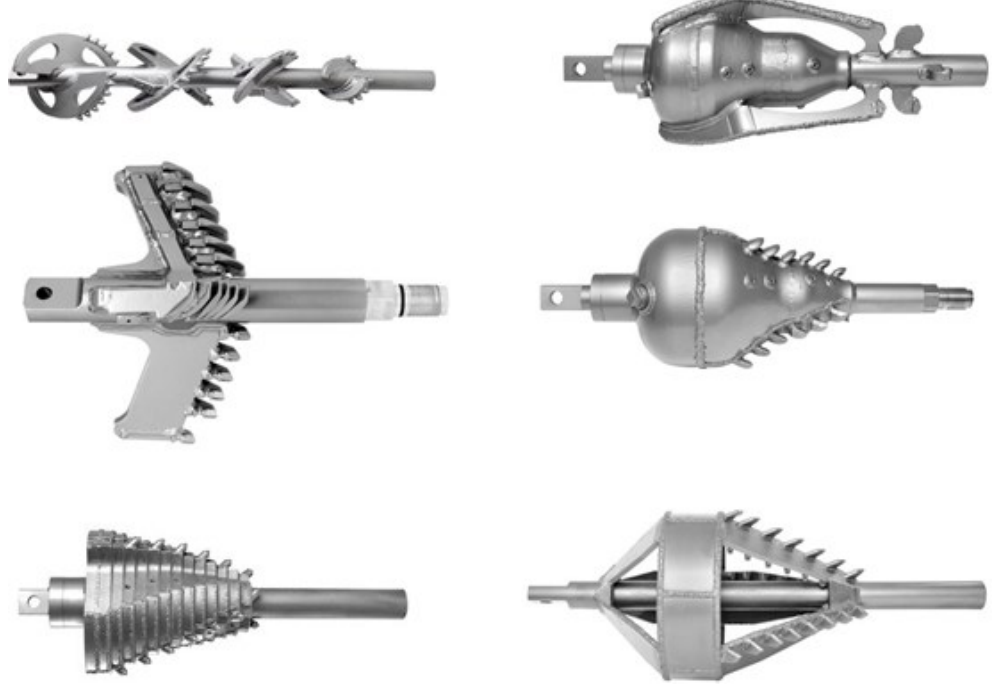

Fig. 2 Different types of reamers

The second step is to pre-ream the pilot hole and enlarge it to a size sufficient to safely install the product lines. A reamer is pulled back and rotated while pumping drilling fluid to cut and remove solids to enlarge the hole. Pre-reaming speeds will vary depending on existing soil conditions and the amount of cuttings that are removed from the hole. Bentonite and other additives will be used to ensure a clean and stable hole. Bentonite is used to create a "cake layer" around the outside of the hole during pre-reaming. This will help with the stability of the bore hole and with fluid loss or infiltration. Additives such as polymers are used to help break up the clay soils. A more evenly mixed drilling fluid will prevent any blockages inside of the bore hole.

The final step is the pullback of the pipe within the pre-reamed hole. The drill rod and reamer will be attached to a swivel, which is utilized between the product line and the reamer to prevent any torsional stress from the rotating drill string being transferred to the product pipe. As the product pipe is pulled into the drill hole, drilling fluid is pumped downhole to provide lubrication to the product pipe (Hadlee \& Brunton, 2019).

\section{APPLICABILITY OF THE HDD TECHNOLOGY}

It is advisable to use it in urban areas with densely populated utilities, where otherwise conventional utilities in the ditch with conventional methods are not 
feasible, very difficult or costly. With this technology, it is possible to pass the pipelines under different objects (for example, highways, rivers, railways). The technology does not require big separate entrance and exit pits. However, a storage pool is required to collect and clean the drilling fluid.

The duration of construction with this technology is significantly shorter than for conventional open trenching. Comparative cost analyses show that most utility construction tasks can be solved at a lower cost by directional drilling than other conventional technologies.

Basically, the performance of the machine determines the bore length and diameter can be achieved with horizontal guided drilling, but the actual result is strongly influenced by the structure of the drilled soil. The theoretical boundaries of technology, the existence of the required special tools and favourable rock conditions:

- available pipe diameter: $\varnothing 1500-1600 \mathrm{~mm}$;

- available drilling length: $\sim 5000 \mathrm{~m}$.

The maximum value of the two features above is not available at the same time. With a length of thousands of meters, the bore diameter is usually less than $1000 \mathrm{~mm}$. Machine performance includes three basic parameters. These are the magnitude of the tensile force applied to the movement of the range of pipes, the amount of torque that can be released by the machine and the range of speeds with which the drill can be operated.

\section{DESCRIPTION OF THE FINITE ELEMENT MODEL}

The design of a reamer should be verified for extreme operating conditions when the tool is subjected by the maximum axial forces and maximum torque limit provided by the engine. In addition, the pressure of the flushing liquid applied should be also taken into account. The pressure generated by the rinse pump can be up to 100 bars which is required to accelerate the flow out of the nozzles of the reamer. The stress contribution caused by these loads is particularly complicated for the barrel-types tools. In this paper a brief description is presented about the modelling assumptions of this drilling equipment. To perform the numerical computation of the reamer, the following geometry is used (Figs. 3 and 4).

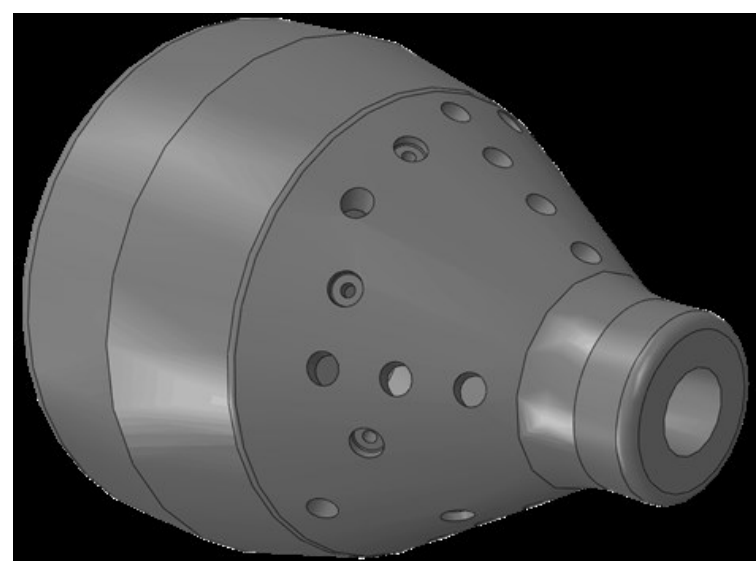

Fig. 3 Geometry of the reamer 


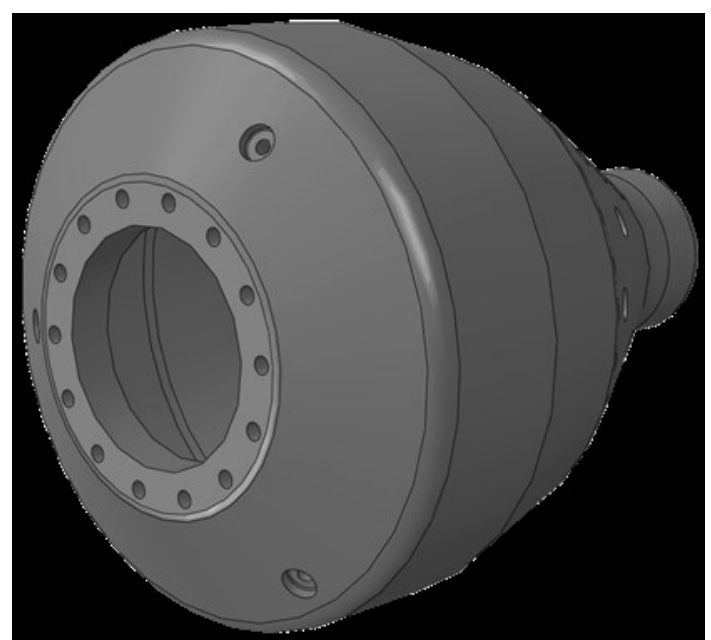

Fig. 4 Back geometry of the reamer

The torque on this tool is computed by assuming that, it arises from the real cutting conditions on the cutting teeth. There is a simplified consideration which assumes some auxiliary points in the FE model (near the cutting teeth) to which concentrated forces are applied. The concentrated forces should be parallel and perpendicular to the drilling axis and so the maximum torque limit is given in the direction of the drilling axis as a torque vector.

The implementation of the auxiliary points for the force system is taken place on the outer surface of the reamer tool based on the midpoints of a planned cutting tooth position as shown on a flat surface of a mantle surface in Fig. 3. In the FE model there are rigid links between the auxiliary points and the node points of bores. The force magnitudes are given from the equilibrium equations $\left(\sum F_{\xi}=0\right.$, $\left.M_{n}=0\right)$ and an associated condition $\left(M_{z}=\right.$ const. $)$ for the torque vector component deriving from the maximum torque limit that take a symmetry plane containing the auxiliary points and perpendicular to the drilling axis. In this case there are no more than three conditions for the parallel forces which can be imposed to give a pure torsion for the tool. As shown in Fig. 5, there are 4 planes (signed with circled numbers) and the first of which is shown in Fig. 6.

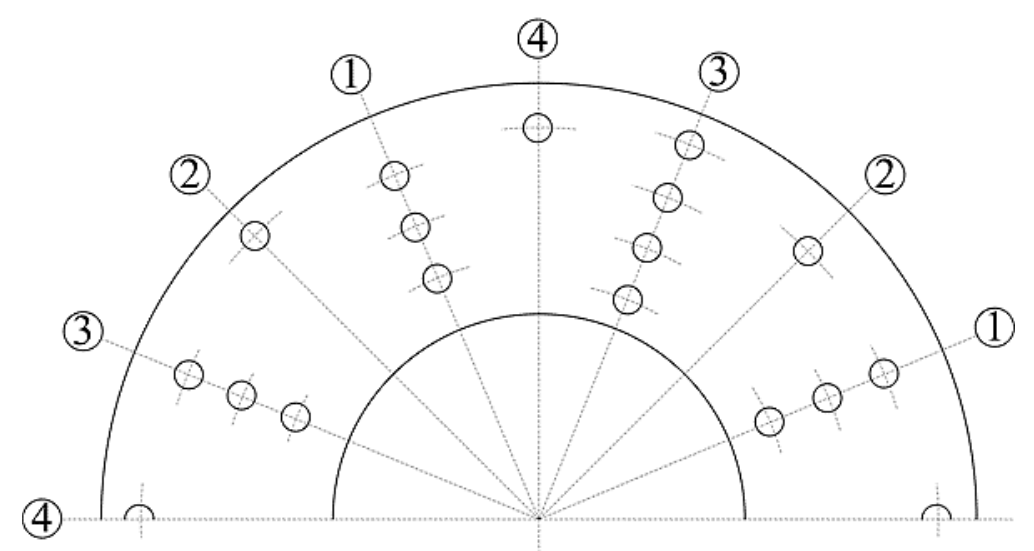

Fig. 5 The bore distribution of the cutting teeth 


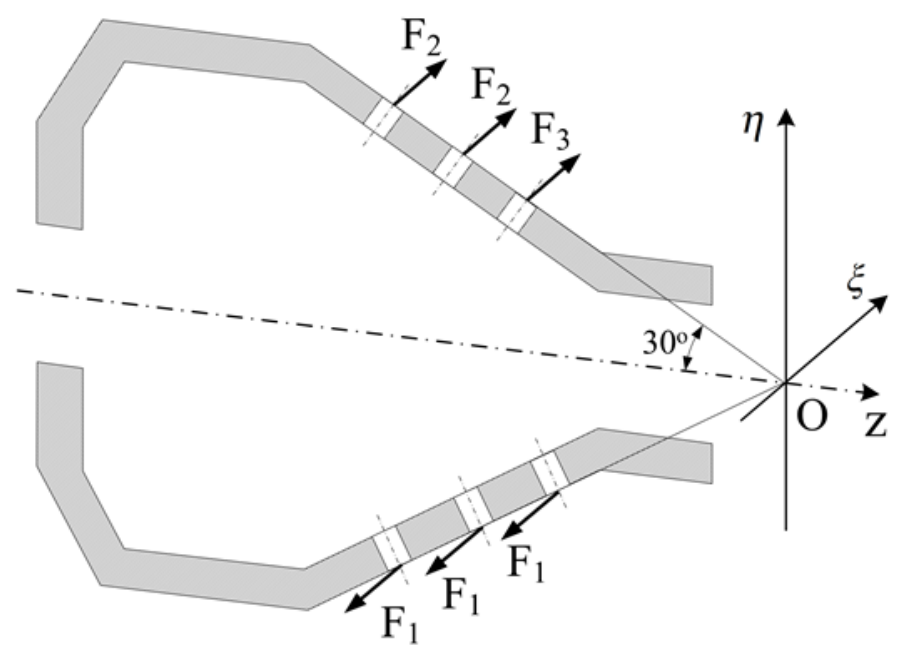

Fig. 6 Possible allocation to the forces on plane 1

For simplicity, the given torque is equally divided between the four planes and unique non-trivial solutions are given for forces in the force system assuming that there are three force magnitudes as unknown in the parallel force system taken by the auxiliary points in each plane. If there are more than three forces acting on the points of the symmetry plane, some equation must be satisfied between force magnitudes. Apparently, the calculated magnitudes vary with the rearrangement of equality between forces. The stress distribution varies locally when the specified force system is changed but the maximum effective stress obtained from FE analyses is hardly changed its effect. Assuming all the nozzles are clogged, the pressure on all the inner surfaces of the tool is 100 bar, which is the maximum pressure generated by the rinse pump. The drilling force is also considered in the FE simulation: The reamer can be under axial loading, therefore, the axial loads acting on the right end of the reamer (Fig. 3) opposite to the fixity applied is directed along the drilling axis. The displacement boundary condition is very simple, all the bores have zero displacements.

In order to investigate the stress distribution on the drilling tool, an accurate finite element mesh is required for the model. The reamer body (Figs 3 and 4) without the cutting teeth and nozzles are meshed and analysed by the FE simulation. More detailed description of finite element modelling can be found in many papers and works such as book of Bathe (Bathe, 1996). Here, a free type finite element mesh is used which contains 10 -nodes tetrahedral continuum elements (C3D10) and the solid structure is subdivided into 844410 pieces (finite elements); it means that, the total number of variables in this model is 3701013. The FE model assumes an elastic material behaviour, which requires the mechanical properties of the steel: the Young's modulus is $2.1 \times 10^{5} \mathrm{MPa}$ and Poisson's ratio is 0.3 . This material behaviour is assumed to be linear elastic until the effective stress reaches proportional limit and in this region the stressstrain relation is represented by Hooke's law. 


\section{ANALYSIS RESULTS}

The numerical simulation always enables different levels of loading. Firstly, only pure torsion is considered on the tool, which comes from the parallel forces (applied at the auxiliary points). As resulted from the numerical simulation, which is shown in Fig. 7, the maximum von Mises stress is about $62.8 \mathrm{MPa}$ and it occurs on the bores of the cutting teeth and the bores of fixing screws. However the stresses decrease quickly below $5 \mathrm{MPa}$ in the remaining part.

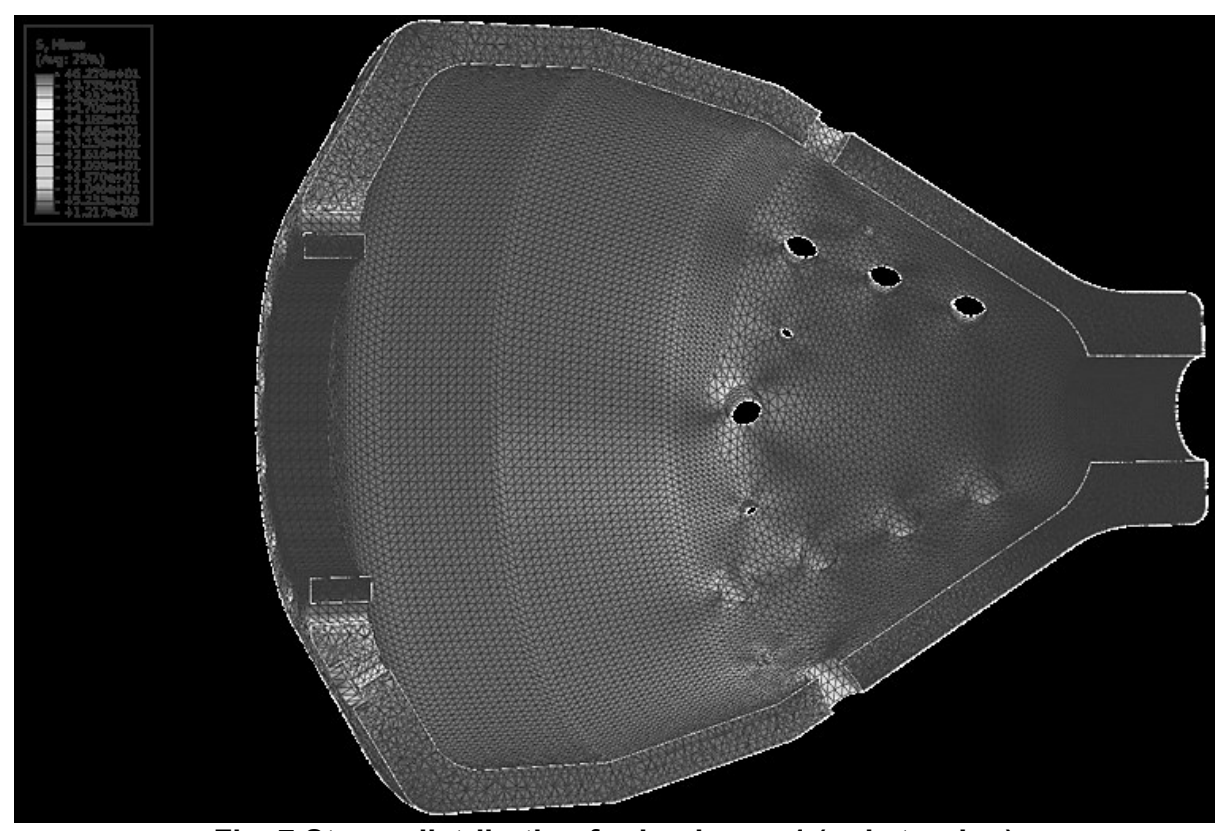

Fig. 7 Stress distribution for load case 1 (only torsion)

In the second load case, the reamer is subjected to internal pressure, axial load and torsion, in the same time. In this load scenario, the maximum Mises stress is resulted about $276 \mathrm{MPa}$ on the bores and the remaining part of the tool shows $92 \mathrm{MPa}$ as is illustrated by Fig. 8.

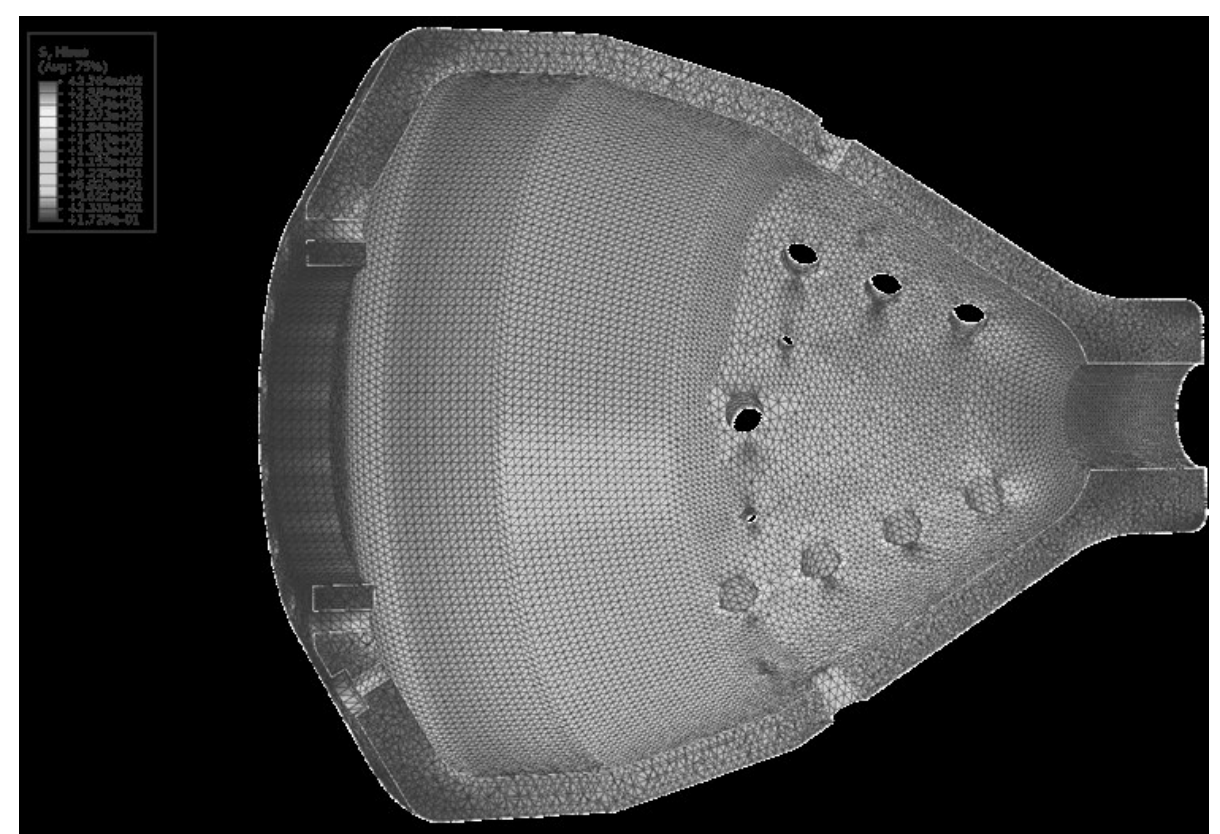

Fig. 8 Stress distribution for load case 2 (internal pressure, torsion and axial load) 
The maximum displacement occurs at the end of the reamer, where the axial load is applied and the maximum displacement magnitude is about $0.075 \mathrm{~mm}$ as is seen on Fig. 9.

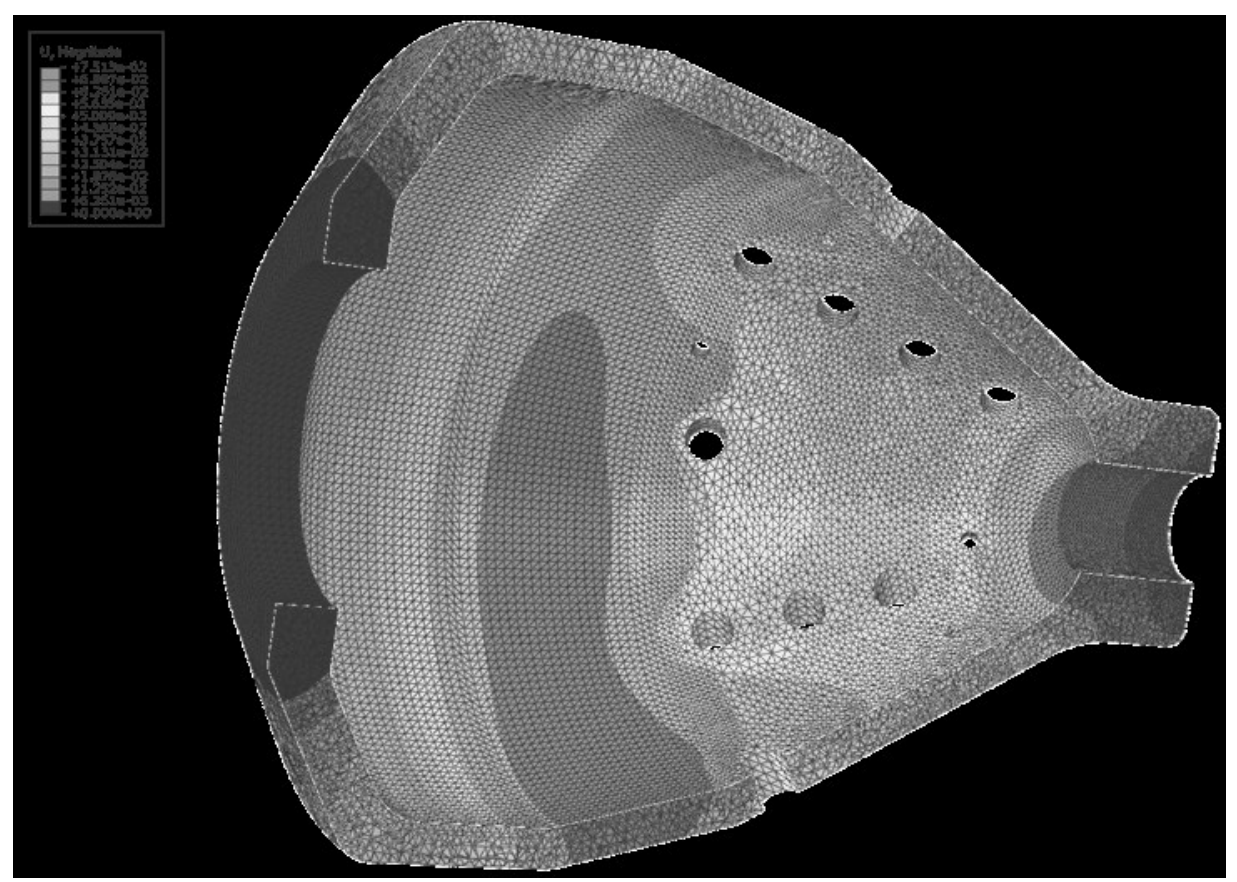

Fig. 9 Displacement distribution for load case 2

\section{CONCLUSIONS}

The work presented in this paper describes the basic assumptions for making numerical simulation for a barrel reamer. To achieve the stress analysis for this problem it advised to use finite element simulation software because of the complex geometry of the drilling equipment and the difficulty in boundary conditions. The numerical example described here gives a brief helpful illustration for analysing these kinds of problems in this field.

\section{ACKNOWLEDGEMENTS}

The described article/presentation/study was carried out as part of the EFOP-3.6.1-162016-00011 "Younger and Renewing University - Innovative Knowledge City institutional development of the University of Miskolc aiming at intelligent specialisation" project implemented in the framework of the Szechenyi 2020 program. The realization of this project is supported by the European Union, co-financed by the European Social Fund.

\section{REFERENCES}

Bathe, K.J. (1996). Finite Element Procedures, Prentice-Hall, Inc., New Jersey,

Hadlee \& Brunton, (2017), What is Horizontal Directional Drilling, Available at: https://www.hadleeandbrunton.co.nz/directional-drilling/what-is-horizontaldirectional-drilling/ [Accessed 20 Apr. 2017].

Hair, J. D. (1991) Analysis of Subsurface Pressures Involved With Directionally Controlled Horizontal Drilling. Proceedings, Pipeline Crossings Specialty Conference, March 25-27, Denver, Colorado. American Society of Civil Engineers, New York. 


\begin{abstract}
.
Horizontal Directional Drilling (HDD) is a method of installing underground pipelines, cables and service conduit through trenchless methods. The tools and techniques used in the HDD process are an outgrowth of the oil well drilling industry, too. Installation of a pipeline by HDD is a three stages process. The first stage consists of directionally drilling a small diameter pilot hole along a designed directional path. Drilling fluid is pumped through the drill pipe to the drill bit where high pressure jets and the bit will grind the soils ahead of the drill stem. The second stage involves enlarging this pilot hole to a diameter suitable for installation of the pipeline. A reamer is pulled back and rotated while pumping drilling fluid to cut and remove solids to enlarge the hole. A kind of reamer is the subject of this paper. The final stage consists of pulling the pipeline back into the pre-reamed hole. The most important part of the mechanical analysis is to define the boundary conditions for operating situations when the tool is under maximum load. In this paper we present a helpful description for giving boundary conditions to perform the numerical simulation of a barrel reamer.
\end{abstract}

Keywords: HDD technology, reamer, FEM, stress, displacement 